ECHER, M.M.; FERNANDES, M.C.A.; RIBEIRO, R.L.D.; PERACCHI, A.L. Avaliação de genótipos de Capsicum para resistência ao ácaro branco. Horticultura Brasileira, Brasília, v. 20, n. 2, p.217-221, junho 2.002.

\title{
Avaliação de genótipos de Capsicum para resistência ao ácaro branco
}

\author{
Márcia M. Echer ${ }^{1}$; Maria C.A. Fernandes ${ }^{2}$; Raul L.D. Ribeiro ${ }^{3}$; Adriano L. Peracchi ${ }^{3}$ \\ ${ }^{2}$ PESAGRO-RIO, Estrada Rio-S.Paulo, km. 47, 23.851-970 Seropédica - RJ; ${ }^{3}$ UFRRJ; E-mail: mmecher@carpa.ciagri.usp.br
}

\section{RESUMO}

Objetivando resistência genética ao ácaro branco (Polyphagotarsonemus latus), foram avaliadas 15 introduções de Capsicum, originárias do Banco de Germoplasma de Hortaliças da UFV, um híbrido e quatro cultivares de pimentão. O estudo foi conduzido em casa de vegetação, sob condições de disseminação natural do ácaro, adotando-se delineamento experimental inteiramente casualizado com seis repetições por genótipo. As avaliações foram procedidas através da contagem do número de ácaros adultos presentes em amostras de folhas e de uma escala de notas referente à severidade dos sintomas. A avaliação por meio de sintomas mostrou-se mais adequada para diferenciar os genótipos em resistentes e suscetíveis. Do material genético avaliado, destacaram-se as introduções BGH/UFV 1774 (C. annuum) e BGH/UFV 5086 (C. frutescens) como resistente $\mathrm{e}$ altamente resistente a Polyphagotarsonemus latus, respectivamente.

Palavras-chave: Polyphagotarsonemus latus, Capsicum annuum, Capsicum frutescens, pimentão.

\begin{abstract}
Evaluation of Capsicum genotypes for resistance to the broad mite

Fifteen Capsicum accessions from the Vegetable Crops Germplasm Bank from the Universidade Federal de Viçosa (Brazil), one hybrid and four pepper cultivars were evaluated in greenhouse for resistance to the broad mite (Polyphagotarsonemus latus). The experiment was set in a completely randomized (split plot in time) design with six replicates per genotype. Evaluations were performed either by counting the number of adult mites present on leaf samples weekly collected or by a grading scale related to symptom severity on infested plants. Symptoms assessment was shown to be more suitable for separating genotypes in resistant or susceptible to the mite. The accessions BGH/UFV 1774 (C. annuum) and BGH/UFV 5086 (C. frutescens) were respectively ranked as resistant and highly resistant to $P$. latus under severe testing conditions.
\end{abstract}

Keywords: Polyphagotarsonemus latus, Capsicum annuum, Capsicum frutescens, pepper.

(Aceito para publicação em 08 de janeiro de 2.002)

$\mathrm{O}$ pimentão (Capsicum annuum L.) é cultivado em todo o território brasileiro, sendo a Região Sudeste a principal produtora, graças aos grandes centros consumidores. Trata-se de uma das 10 hortaliças de maior importância econômica no mercado hortifrutigranjeiro nacional, sendo entretanto bastante exigente em tratos culturais e muitas vezes, prejudicada pelo ataque de pragas. Dentre elas, destaca-se o ácaro branco [Polyphagotarsonemus latus (Banks) (Acari: Tarsonemidae)], que tem se tornado alvo de atenção devido à sua freqüente e severa ocorrência em diversas áreas produtoras. $\mathrm{O}$ ácaro branco está distribuído nos trópicos e também em regiões de clima temperado, tendo sido registrado em amplo espectro de espécies agrícolas, ornamentais e silvestres (Jeppson et al., 1975; Brown \& Jones, 1983; Li et al., 1985; Gerson, 1992; Fan \& Pettit, 1994). Em pimentão, sua incidência já foi assinalada em praticamente todo o mundo (Hambleton, 1938; Cross, 1979; Li et al., 1985; Gerson, 1986; David, 1986; Roditakis \& Drossos, 1987).
Populações elevadas de P. latus surgem da combinação de temperatura e umidade alta, com baixa luminosidade (Flechtmann, 1967). Parra (1968) relatou que a multiplicação de $P$. latus é favorecida em microclimas úmidos e sob insolação moderada. Gallo et al. (1988) afirmaram que o ácaro branco causa danos significativos quando ocorrem condições climáticas favoráveis, como temperatura e umidade relativa do ar elevadas. Por outro lado o desenvolvimento de P. latus em algodoeiro depende mais da idade da planta do que das condições de temperatura e umidade prevalecentes (Chiavegato,1975). Li et al. (1985) estudaram os efeitos de temperatura e umidade na incidência de $P$. latus em feijoeiro, concluindo que este último fator tem influência na colonização pela praga, porém não tão significativa quanto a temperatura.

Danos causados por P. latus no desenvolvimento vegetativo de muitas espécies de interesse econômico têm significado relevante em muitas regiões (Peña \& Bullock, 1994). O ácaro prefere se alimentar na superfície abaxial das folhas, que, consequentemente, se tor- nam curvadas para baixo, encarquilhadas, ressecadas e bronzeadas, podendo chegar a cair prematuramente (Smith, 1939 citado por Gerson, 1992). Geralmente, as folhas novas são mais intensamente infestadas, tornando-se murchas e freqüentemente necrosando, assim como as gemas apicais (Oliveira, 1972).

Os sintomas ocasionados por $P$. latus diferem em função do hospedeiro, refletindo reações específicas das plantas à alimentação da praga e a supostas toxinas. Alguns desses sintomas já foram confundidos com doenças (Jeppson et al., 1975), injúrias de herbicidas (Gerson, 1992) e, até mesmo, deficiência nutricional (Cross \& Bassett, 1982; Gerson, 1992). Bassett (1981) afirmou que a manifestação dos sintomas em certas espécies hospedeiras pode ser muito rápida, indicando que poucos ácaros presentes na planta são suficientes para provocar prejuízos econômicos. Injúrias causadas pelo parasitismo de $P$. latus têm sido avaliadas por meio de um sistema de classificação visual (Oliveira \& Calcagnolo, 1974; Schoonhoven et al., 1978; Peña, 1990; Peña \& Bullock, 1994)

\footnotetext{
${ }^{1}$ Parte da tese de mestrado do primeiro autor, apresentada ao Depto. de Fitotecnia da UFRRJ.
} 
A aplicação de pesticidas tem sido o único método de controle ao ácaro branco (Gerson, 1992). De acordo com David (1986) e Rao \& Ahmed (1983 e 1986), pulverizações foliares com determinados inseticidas influenciam a ressurgência de $P$. latus em pimentão. A provável razão dessa ressurgência seria a eliminação de predadores e parasitóides, ou o estimulo à reprodução do ácaro por doses sub-letais de produtos químicos. Assim, a utilização de cultivares geneticamente resistentes seria ideal, desde que populações da praga pudessem ser reduzidas a níveis satisfatórios, sem onerar a produção. Além disso, a resistência pode ser associada a praticamente todos os outros métodos de controle (Vendramim, 1984; Lara, 1991). No Brasil, acredita-se que pesquisas nesse campo do melhoramento vegetal deverão assumir destaque e vir a contribuir para o controle de importantes pragas de hortaliças (Villas Bôas, 1989).

Resistência a $P$. latus já foi detectada em uma espécie selvagem de batata (Solanum berthaultii) por Gibson \& Valencia (1978). Em pimentão (Capsicum annuum), três linhagens de duplo-haplóide (HD60, HD16 e HD12) foram consideradas tolerantes (Depestre \& Gomez , 1995). Nagai \& Lourenção (1987) selecionaram plantas de pimenteira "Cambuci" (Capsicum baccatum) resistentes ao ácaro branco. Esse genótipo deu origem à cultivar IACUbatuba, igualmente resistente à praga .

Este trabalho teve por objetivo avaliar genótipos de Capsicum para resistência ao ácaro branco.

\section{MATERIAL E MÉTODOS}

O estudo foi conduzido em casa de vegetação da PESAGRO-RIO. Foram avaliados 15 genótipos de Capsicum provenientes do Banco de Germoplasma de Hortaliças da UFV (C. annuum BGH/ UFV 58; 88; 106; 421; 1465; 1774; $2850 ; 2875 ; 2901 ; 3023 ; 3034 ; 3062$; 3071 e 3077 ; C. frutescens BGH/UFV 5086), além do híbrido Magali e quatro cultivares de pimentão, de uso corriqueiro: Cascadura Ikeda, Myr-10, Myr-29 e Margareth. A cultivar Cascadura Ikeda (CI) foi utilizada como padrão de suscetibilidade ao ácaro, funcionando também como foco de disseminação natural da praga. Para isto, a cultivar CI foi semeada aproximadamente um mês antes dos demais genótipos.

A semeadura foi realizada em bandejas de isopor de 128 células, contendo substrato comercial. Após 30 dias, as mudas foram transplantadas para vasos contendo $3,5 \mathrm{~kg}$ de solo autoclavado e adubado com NPK (4-14-8) e esterco bovino, mantendo-se duas plantas por vaso. Nessa ocasião, as plantas da cultivar CI apresentavam em média 15,1 ácaros adultos por folha.

A avaliação do comportamento dos genótipos foi baseada na severidade dos sintomas e no número de ácaros $/ \mathrm{cm}^{2} \mathrm{de}$ área foliar. As leituras foram iniciadas no $25^{\circ}$ dia após o transplante, quando surgiram os primeiros sintomas, tendo, a partir daí freqüência semanal. Para contagem dos ácaros, coletou-se uma folha por vaso, correspondendo à região apical das plantas. As amostras foram acondicionadas em sacos de papel e devidamente etiquetadas. No laboratório, procedeu-se à contagem das formas adultas de $P$. latus com auxílio de um estereomicroscópio, sob magnificação de 20 vezes, numa área circular de 1,13 $\mathrm{cm}^{2}$ da face abaxial, próxima à inserção do pecíolo. As coletas foram realizadas durante cinco semanas, ocasião em que foram paralizadas em função do intenso desfolhamento que ocorreu em alguns dos genótipos.

Para estimar a intensidade dos sintomas, adaptou-se a escala de notas de Oliveira \& Calcagnolo (1974) desenvolvida para a cultura do algodoeiro. Esta escala adaptada apresentou limites de 0 a 4 (nota 0 = folhas não danificadas; nota 1 = folhas de consistência normal, mas com ligeiras ondulações e bordos voltados para cima ou para baixo; nota $2=$ folhas pouco coriáceas e arqueadas para baixo, deformadas, tendo ou não superfície abaxial de aspecto bronzeado; nota 3 = folhas coriáceas e arqueadas para baixo, deformadas, mostrando superfície abaxial de aspecto bronzeado, em plantas formando tufos de folhas diminutas; nota 4 = frutos retorcidos, com casca áspera e bronzeada, ausência de botões florais, plantas desfolhadas e com brotações abortadas).

$\mathrm{O}$ ensaio foi conduzido em delineamento inteiramente casualizado com subparcelas distribuídas no tempo ("split plot in time") e seis repetições por genótipo. Os dados obtidos foram transformados em $\log (x+0,5)$, sendo $x$ equivalente ao número de ácaros de cada contagem. Após a transformação, os dados foram submetidos à análise de variância e as médias comparadas por meio da análise de agrupamento, a 5\% de probabilidade, segundo o método de Scott \& Knott (1974). Buscou-se ainda correlacionar severidade de sintomas com níveis populacionais do ácaro presentes nas amostras foliares.

\section{RESULTADOS E DISCUSSÃO}

As análises de variância revelaram diferenças altamente significativas, evidenciando considerável variabilidade entre os genótipos. Observou-se interação significativa entre genótipos e épocas de amostragem. O comportamento dos acessos de Capsicum em relação ao ácaro branco, avaliado através da escala de notas ou do número de ácaros/ $\mathrm{cm}^{2}$ de área foliar, está configurado nas Tabelas 1 e 2, respectivamente.

Aos 25 dias pós transplantio (DAT), a classificação dos genótipos por meio da escala de notas traduziu-se em quatro grupos. No primeiro, ficaram incluídas as introduções BGH/UFV 2901 e 3071, que apresentaram as notas mais elevadas (2,2 e 1,7); no segundo, BGH/UFV 88; 58; 106; 3034; Myr-10 e Cascadura Ikeda, cujas notas variaram de 1,0 a 1,3; no terceiro, situaram-se BGH/UFV 421; 1465; 2850; 3023; 3062; 3077; Margareth e Myr-29, com notas de 0,5 a 0,8; finalmente no quarto grupos, foram enquadradas BGH/UFV 1774; 5086; 2875 e Magali, que se apresentaram como os mais resistentes a $P$. latus, com notas variando de 0,0 a 0,2 . As introduções $\mathrm{BGH} /$ UFV 1774 e 5086, nessa ocasião, não apresentaram quaisquer sinais de injúrias causadas pelo ácaro, mas não diferiram estatisticamente do híbrido Magali nem da introdução BGH/UFV 2875, levemente danificadas, com elas constituindo um único grupos.

Quando se procedeu à avaliação através da contagem do número de ácaros por área foliar, os genótipos ficaram separados em apenas dois grupos. Apesar de ter havido correlação significativa ( $1 \%$ de probabilidade pelo teste " $t$ ") entre os dois métodos de avaliação, esta foi muito baixa $(-0,098)$, indicando a im- 
Avaliação de genótipos de Capsicum para resistência ao ácaro branco.

Tabela 1. Comportamento de genótipos de Capsicum, de acordo com a severidade de sintomas ( $0=$ sem sintomas; $4=$ sintomas severos) induzidos pelo ácaro branco (Polyphagotarsonemus latus), sob condições de casa de vegetação. Seropédica, PESAGRO, 1998.

\begin{tabular}{|c|c|c|c|c|c|c|c|c|c|}
\hline Genótipo ${ }^{1}$ & $25 \mathrm{DAT}^{2}$ & Genótipo & 32 DAT & Genótipo & 39 DAT & Genótipo & 46 DAT & Genótipo & 53 DAT \\
\hline BGH 2901 & $2,2 \mathrm{~A} 3$ & BGH 2901 & $3,0 \mathrm{~A}$ & C. Ikeda & $3,0 \mathrm{~A}$ & BGH 3023 & $3,8 \mathrm{~A}$ & BGH 421 & $3,8 \mathrm{~A}$ \\
\hline BGH 3071 & $1,7 \mathrm{~A}$ & BGH 3071 & $2,8 \mathrm{~A}$ & BGH 3071 & $3,0 \mathrm{~A}$ & BGH 2901 & $3,2 \mathrm{~A}$ & BGH 88 & $3,7 \mathrm{~A}$ \\
\hline BGH 106 & $1,3 \mathrm{~B}$ & BGH 58 & $2,7 \mathrm{~A}$ & BGH 3034 & $3,0 \mathrm{~A}$ & BGH 88 & $3,2 \mathrm{~A}$ & Myr-10 & $3,5 \mathrm{~A}$ \\
\hline C. Ikeda & $1,2 \mathrm{~B}$ & BGH 3034 & $2,5 \mathrm{~A}$ & BGH 2901 & $3,0 \mathrm{~A}$ & C. Ikeda & $3,0 \mathrm{~A}$ & BGH 2901 & $3,5 \mathrm{~A}$ \\
\hline BGH 3034 & $1,2 \mathrm{~B}$ & BGH 3023 & $2,5 \mathrm{~A}$ & BGH 58 & $3,0 \mathrm{~A}$ & Magali & $3,0 \mathrm{~A}$ & Margareth & $3,5 \mathrm{~A}$ \\
\hline BGH 58 & $1,2 \mathrm{~B}$ & BGH 106 & $2,3 \mathrm{~A}$ & BGH 106 & $2,8 \mathrm{~A}$ & Margareth & $3,0 \mathrm{~A}$ & Magali & $3,3 \mathrm{~A}$ \\
\hline Myr-10 & $1,0 \mathrm{~B}$ & BGH 2850 & $2,3 \mathrm{~A}$ & BGH 88 & $2,8 \mathrm{~A}$ & BGH 3071 & $3,0 \mathrm{~A}$ & BGH 3062 & $3,3 \mathrm{~A}$ \\
\hline BGH 88 & $1,0 \mathrm{~B}$ & C. Ikeda & $2,2 \mathrm{~B}$ & Margareth & $2,7 \mathrm{~A}$ & BGH 106 & $3,0 \mathrm{~A}$ & BGH 3023 & $3,3 \mathrm{~A}$ \\
\hline BGH 3062 & $0,8 \mathrm{C}$ & BGH 421 & $2,0 \mathrm{~B}$ & BGH 3023 & $2,7 \mathrm{~A}$ & BGH 3062 & $3,0 \mathrm{~A}$ & BGH 58 & $3,3 \mathrm{~A}$ \\
\hline BGH 2850 & $0,8 \mathrm{C}$ & BGH 3062 & $2,0 \mathrm{~B}$ & Magali & $2,5 \mathrm{~A}$ & BGH 3034 & $3,0 \mathrm{~A}$ & C. Ikeda & $3,2 \mathrm{~B}$ \\
\hline Myr-29 & $0,7 \mathrm{C}$ & BGH 88 & $2,0 \mathrm{~B}$ & BGH 3077 & $2,5 \mathrm{~A}$ & BGH 58 & $3,0 \mathrm{~A}$ & BGH 106 & $3,2 \mathrm{~B}$ \\
\hline BGH 421 & $0,7 \mathrm{C}$ & Magali & $1,8 \mathrm{~B}$ & BGH 3062 & $2,5 \mathrm{~A}$ & Myr-29 & $2,8 \mathrm{~A}$ & BGH 2850 & $3,2 \mathrm{~B}$ \\
\hline BGH 3077 & $0,7 \mathrm{C}$ & BGH 3077 & $1,8 \mathrm{~B}$ & BGH 2875 & $2,5 \mathrm{~A}$ & BGH 421 & $2,7 \mathrm{~A}$ & Myr-29 & $3,0 \mathrm{~B}$ \\
\hline BGH 3023 & $0,7 \mathrm{C}$ & Margareth & $1,7 \mathrm{C}$ & Myr-29 & $2,3 \mathrm{~A}$ & BGH 3077 & $2,7 \mathrm{~A}$ & BGH 3077 & $3,0 \mathrm{~B}$ \\
\hline BGH 1465 & $0,7 \mathrm{C}$ & BGH 1465 & $1,5 \mathrm{C}$ & BGH 421 & $2,3 \mathrm{~A}$ & BGH 2875 & $2,7 \mathrm{~A}$ & BGH 3071 & $3,0 \mathrm{~B}$ \\
\hline Margareth & $0,5 \mathrm{C}$ & Myr-10 & $1,3 \mathrm{C}$ & BGH 2850 & $2,3 \mathrm{~A}$ & BGH 2850 & $2,7 \mathrm{~A}$ & BGH 3034 & $3,0 \mathrm{~B}$ \\
\hline Magali & $0,2 \mathrm{D}$ & Myr-29 & $1,3 \mathrm{C}$ & Myr-10 & $2,2 \mathrm{~A}$ & Myr-10 & $2,3 \mathrm{~A}$ & BGH 2875 & $3,0 \mathrm{~B}$ \\
\hline BGH 2875 & $0,2 \mathrm{D}$ & BGH 2875 & $1,3 \mathrm{C}$ & BGH 1465 & $1,7 \mathrm{~B}$ & BGH 1465 & $2,0 \mathrm{~A}$ & BGH 1465 & $2,3 \mathrm{~B}$ \\
\hline BGH 5086 & $0,0 \mathrm{D}$ & BGH 1774 & $1,0 \mathrm{C}$ & BGH 1774 & $1,5 \mathrm{~B}$ & BGH 1774 & $1,7 \mathrm{~B}$ & BGH 1774 & $1,7 \mathrm{C}$ \\
\hline BGH 1774 & $0,0 \mathrm{D}$ & BGH 5086 & $0,2 \mathrm{D}$ & BGH 5086 & $0,2 \mathrm{C}$ & BGH 5086 & $0,2 \mathrm{C}$ & BGH 5086 & $0,2 \mathrm{D}$ \\
\hline Genótipo & 60 DAT & Genótipo & 67 DAT & Genótipo & 74 DAT & Genótipo & 81 DAT & Genótipo & 88 DAT \\
\hline BGH 421 & $3,8 \mathrm{~A}$ & BGH 88 & $3,9 \mathrm{~A}$ & Myr-10 & $4,0 \mathrm{~A}$ & Myr-10 & $4,0 \mathrm{~A}$ & Myr-10 & $4,0 \mathrm{~A}$ \\
\hline Magali & $3,7 \mathrm{~A}$ & BGH 421 & $3,8 \mathrm{~A}$ & Myr-29 & $4,0 \mathrm{~A}$ & Myr-29 & $4,0 \mathrm{~A}$ & Myr-29 & $4,0 \mathrm{~A}$ \\
\hline BGH 3062 & $3,7 \mathrm{~A}$ & BGH 3062 & $3,8 \mathrm{~A}$ & Magali & $4,0 \mathrm{~A}$ & Magali & $4,0 \mathrm{~A}$ & Magali & $4,0 \mathrm{~A}$ \\
\hline BGH 2901 & $3,7 \mathrm{~A}$ & BGH 2901 & $3,8 \mathrm{~A}$ & BGH 421 & $4,0 \mathrm{~A}$ & Margareth & $4,0 \mathrm{~A}$ & Margareth & $4,0 \mathrm{~A}$ \\
\hline BGH 88 & $3,7 \mathrm{~A}$ & Magali & $3,7 \mathrm{~A}$ & BGH 3062 & $4,0 \mathrm{~A}$ & BGH 421 & $4,0 \mathrm{~A}$ & BGH 421 & $4,0 \mathrm{~A}$ \\
\hline Margareth & $3,5 \mathrm{~A}$ & Margareth & $3,7 \mathrm{~A}$ & BGH 2901 & $4,0 \mathrm{~A}$ & BGH 3062 & $4,0 \mathrm{~A}$ & BGH 3071 & $4,0 \mathrm{~A}$ \\
\hline BGH 2850 & $3,5 \mathrm{~A}$ & Myr-10 & $3,5 \mathrm{~A}$ & BGH 1465 & $4,0 \mathrm{~A}$ & BGH 2901 & $4,0 \mathrm{~A}$ & BGH 3062 & $4,0 \mathrm{~A}$ \\
\hline Myr-10 & $3,3 \mathrm{~A}$ & Myr-29 & $3,5 \mathrm{~A}$ & C. Ikeda & $3,8 \mathrm{~A}$ & BGH 3023 & $4,0 \mathrm{~A}$ & BGH 3034 & $4,0 \mathrm{~A}$ \\
\hline BGH 106 & $3,3 \mathrm{~A}$ & BGH 106 & $3,5 \mathrm{~A}$ & Margareth & $3,8 \mathrm{~A}$ & BGH 58 & $4,0 \mathrm{~A}$ & BGH 2901 & $4,0 \mathrm{~A}$ \\
\hline BGH 3023 & $3,3 \mathrm{~A}$ & BGH 3023 & $3,5 \mathrm{~A}$ & BGH 3071 & $3,8 \mathrm{~A}$ & BGH 88 & $4,0 \mathrm{~A}$ & BGH 3023 & $4,0 \mathrm{~A}$ \\
\hline BGH 58 & $3,3 \mathrm{~A}$ & BGH 58 & $3,5 \mathrm{~A}$ & BGH 88 & $3,8 \mathrm{~A}$ & BGH 1465 & $4,0 \mathrm{~A}$ & BGH 58 & $4,0 \mathrm{~A}$ \\
\hline C. Ikeda & $3,2 \mathrm{~A}$ & BGH 2850 & $3,5 \mathrm{~A}$ & BGH 3023 & $3,7 \mathrm{~A}$ & C. Ikeda & $3,8 \mathrm{~A}$ & BGH 88 & $4,0 \mathrm{~A}$ \\
\hline Myr-29 & $3,2 \mathrm{~A}$ & C. Ikeda & $3,3 \mathrm{~A}$ & BGH 2850 & $3,7 \mathrm{~A}$ & BGH 3071 & $3,8 \mathrm{~A}$ & BGH 1465 & $4,0 \mathrm{~A}$ \\
\hline BGH 3071 & $3,2 \mathrm{~A}$ & BGH 3071 & $3,3 \mathrm{~A}$ & BGH 106 & $3,5 \mathrm{~B}$ & BGH 106 & $3,8 \mathrm{~A}$ & C. Ikeda & $3,8 \mathrm{~A}$ \\
\hline BGH 3034 & $3,2 \mathrm{~A}$ & BGH 3034 & $3,2 \mathrm{~A}$ & BGH 58 & $3,5 \mathrm{~B}$ & BGH 2875 & $3,8 \mathrm{~A}$ & BGH 106 & $3,8 \mathrm{~A}$ \\
\hline BGH 3077 & $3,0 \mathrm{~A}$ & BGH 3077 & $3,0 \mathrm{~A}$ & BGH 3034 & $3,3 \mathrm{~B}$ & BGH 2850 & $3,8 \mathrm{~A}$ & BGH 2875 & $3,8 \mathrm{~A}$ \\
\hline BGH 2875 & $3,0 \mathrm{~A}$ & BGH 2875 & $3,0 \mathrm{~A}$ & BGH 2875 & $3,3 \mathrm{~B}$ & BGH 3034 & $3,7 \mathrm{~A}$ & BGH 2850 & $3,8 \mathrm{~A}$ \\
\hline BGH 1465 & $2,8 \mathrm{~A}$ & BGH 1465 & $3,0 \mathrm{~A}$ & BGH 3077 & $3,0 \mathrm{~B}$ & BGH 1774 & $3,5 \mathrm{~A}$ & BGH 1774 & $3,5 \mathrm{~A}$ \\
\hline BGH 1774 & $2,0 \mathrm{~B}$ & BGH 1774 & $2,0 \mathrm{~B}$ & BGH 1774 & $2,7 \mathrm{~B}$ & BGH 3077 & $3,3 \mathrm{~A}$ & BGH 3077 & $3,3 \mathrm{~A}$ \\
\hline BGH 5086 & $0,2 \mathrm{C}$ & BGH 5086 & $0,3 \mathrm{C}$ & BGH 5086 & $0,3 \mathrm{C}$ & BGH 5086 & $0,7 \mathrm{~B}$ & BGH 5086 & $1,0 \mathrm{~B}$ \\
\hline
\end{tabular}

${ }^{1}$ Todos os genótipos pertencem à espécie $C$. annuum, com exceção de BGH/UFV 5086 (C. frutescens); BGH/UFV= Banco de Germoplasma de Hortaliças/Universidade Federal de Viçosa; ${ }^{2} \mathrm{DAT}=$ Dias após transplantio das mudas para vasos na casa de vegetação; ${ }^{3}$ Os valores representam médias de seis repetições. Médias seguidas de mesma letra nas colunas, não diferem entre si, pelo teste agrupamento de $\operatorname{Scott} \& \operatorname{Knott} . \mathrm{CV}(\%)=19,21$

portância que representa o método utilizado na seleção de genótipos de Capsicum para resistência ao ácaro branco.
Na segunda avaliação, com 32 DAT, os genótipos formaram quatro e três grupos quanto à severidade de sintomas e presença de ácaros adultos, respectivamente. A introdução BGH/UFV 5086 foi classificada como a mais resistente 
Tabela 2. Número de ácaros brancos (Polyphagotarsonemus latus) por $\mathrm{cm}^{2}$ de área foliar de genótipos de Capsicum, sob condições de casa de vegetação. Seropédica, PESAGRO, 1998.

\begin{tabular}{|c|c|c|c|c|c|c|c|c|c|}
\hline Genótipo 1 & 25 DAT $^{2}$ & Genótipo & 32 DAT & Genótipo & 39 DAT & Genótipo & 46 DAT & Genótipo & 53 DAT \\
\hline BGH 2901 & $25,2 \mathrm{~A} 3$ & Magali & $32,2 \mathrm{~A}$ & Margareth & $5,2 \mathrm{~A}$ & Myr-29 & $26,5 \mathrm{~A}$ & Myr-29 & $15,0 \mathrm{~A}$ \\
\hline BGH 3023 & $21,7 \mathrm{~A}$ & C. Ikeda & $29,0 \mathrm{~A}$ & C. Ikeda & $4,2 \mathrm{~A}$ & Margareth & $17,3 \mathrm{~A}$ & BGH 106 & $7,3 \mathrm{~A}$ \\
\hline BGH 3071 & $18,3 \mathrm{~A}$ & BGH 3062 & $26,5 \mathrm{~A}$ & Myr-10 & $4,2 \mathrm{~A}$ & C. Ikeda & $12,5 \mathrm{~A}$ & BGH 88 & $7,3 \mathrm{~A}$ \\
\hline Magali & $17,2 \mathrm{~A}$ & Myr-10 & $25,7 \mathrm{~A}$ & BGH 2875 & $3,5 \mathrm{~A}$ & BGH 106 & $11,0 \mathrm{~A}$ & C. Ikeda & $6,5 \mathrm{~A}$ \\
\hline BGH 3034 & $16,2 \mathrm{~A}$ & BGH 106 & $23,5 \mathrm{~B}$ & BGH 2850 & $3,3 \mathrm{~A}$ & BGH 58 & $9,8 \mathrm{~A}$ & BGH 3071 & $6,2 \mathrm{~A}$ \\
\hline BGH 3062 & $15,8 \mathrm{~A}$ & BGH 3034 & $21,2 \mathrm{~B}$ & Magali & $2,8 \mathrm{~B}$ & BGH 3062 & $8,3 \mathrm{~B}$ & BGH 3034 & $5,8 \mathrm{~A}$ \\
\hline BGH 88 & $15,5 \mathrm{~A}$ & BGH 2850 & $18,0 \mathrm{~B}$ & Myr-29 & $2,8 \mathrm{~B}$ & BGH 3034 & $7,8 \mathrm{~B}$ & Margareth & $5,2 \mathrm{~A}$ \\
\hline BGH 58 & $14,5 \mathrm{~A}$ & BGH 421 & $17,3 \mathrm{~B}$ & BGH 2901 & $1,8 \mathrm{~B}$ & Myr-10 & $7,5 \mathrm{~B}$ & Myr-10 & $5,2 \mathrm{~A}$ \\
\hline BGH 421 & $14,2 \mathrm{~A}$ & BGH 3071 & $16,7 \mathrm{~B}$ & BGH 421 & $1,7 \mathrm{~B}$ & BGH 1774 & $7,0 \mathrm{~B}$ & BGH 3023 & $5,0 \mathrm{~A}$ \\
\hline C. Ikeda & $13,7 \mathrm{~B}$ & Margareth & $16,0 \mathrm{~B}$ & BGH 3077 & $1,7 \mathrm{~B}$ & BGH 3071 & $6,5 \mathrm{~B}$ & BGH 2901 & $4,7 \mathrm{~A}$ \\
\hline Myr-10 & $12,2 \mathrm{~B}$ & BGH 2875 & $13,8 \mathrm{~B}$ & BGH 3023 & $1,5 \mathrm{~B}$ & Magali & $5,7 \mathrm{~B}$ & BGH 1465 & $4,7 \mathrm{~A}$ \\
\hline BGH 3077 & $9,7 \mathrm{~B}$ & BGH 3077 & $13,0 \mathrm{~B}$ & BGH 106 & $1,3 \mathrm{~B}$ & BGH 2875 & $5,7 \mathrm{~B}$ & BGH 2850 & $4,3 \mathrm{~B}$ \\
\hline BGH 106 & $8,7 \mathrm{~B}$ & BGH 2901 & $12,7 \mathrm{~B}$ & BGH 58 & $1,3 \mathrm{~B}$ & BGH 2850 & $5,0 \mathrm{~B}$ & BGH 58 & $3,7 \mathrm{~B}$ \\
\hline BGH 2875 & $8,7 \mathrm{~B}$ & BGH 3023 & $11,5 \mathrm{~B}$ & BGH 5086 & $1,3 \mathrm{~B}$ & BGH 421 & $4,3 \mathrm{~B}$ & Magali & $3,3 \mathrm{~B}$ \\
\hline BGH 1774 & $8,3 \mathrm{~B}$ & BGH 1774 & $11,5 \mathrm{~B}$ & BGH 1465 & $1,3 \mathrm{~B}$ & BGH 3023 & $4,3 \mathrm{~B}$ & BGH 2875 & $3,3 \mathrm{~B}$ \\
\hline BGH 1465 & $8,2 \mathrm{~B}$ & Myr-29 & $9,8 \mathrm{C}$ & BGH 3071 & $1,2 \mathrm{~B}$ & BGH 1465 & $4,2 \mathrm{~B}$ & BGH 3062 & $2,8 \mathrm{~B}$ \\
\hline BGH 2850 & $7,0 \mathrm{~B}$ & BGH 1465 & $8,5 \mathrm{C}$ & BGH 3062 & $1,2 \mathrm{~B}$ & BGH 2901 & $3,8 \mathrm{~B}$ & BGH 421 & $2,3 \mathrm{~B}$ \\
\hline Margareth & $6,8 \mathrm{~B}$ & BGH 88 & $6,8 \mathrm{C}$ & BGH 88 & $0,7 \mathrm{~B}$ & BGH 88 & $3,3 \mathrm{~B}$ & BGH 5086 & $2,0 \mathrm{~B}$ \\
\hline BGH 5086 & $4,8 \mathrm{~B}$ & BGH 58 & $6,2 \mathrm{C}$ & BGH 1774 & $0,7 \mathrm{~B}$ & BGH 3077 & $2,7 \mathrm{~B}$ & BGH 3077 & $1,8 \mathrm{~B}$ \\
\hline Myr-29 & $2,7 \mathrm{~B}$ & BGH 5086 & $4,8 \mathrm{C}$ & BGH 3034 & $0,3 \mathrm{~B}$ & BGH 5086 & $1,7 \mathrm{~B}$ & BGH 1774 & $1,8 \mathrm{~B}$ \\
\hline
\end{tabular}

${ }^{1}$ Todos os genótipos pertencem à espécie $C$. annuum, com exceção da BGH/UFV 5086 (C. frutescens); BGH/UFV= Banco de Germoplasma de Hortaliças/Universidade Federal de Viçosa; ${ }^{2} \mathrm{DAT}=$ dias após transplantio das mudas para vasos na casa de vegetação; ${ }^{3}$ os valores representam médias de seis repetições; médias seguidas de mesma letra, nas colunas, não diferem entre si, a $5 \%$ de probabilidade, pelo teste de Scott \& Knott; CV $(\%)=38$,

ao ácaro branco quando avaliada por sintomas, apresentando nota média de 0,17 e também revelando o menor número de ácaros presentes. Porém, não diferiu, nessa amostragem, de Myr-29, BGH/UFV 58; 88 e 1465, quanto ao número de ácaros. A introdução BGH/ UFV 1774 também apresentou pouca intensidade de dano, mas não diferiu dos genótipos BGH/UFV 2875, 1465, Myr29, Myr-10 e Margareth. Nessa segunda época de avaliação, vários dos materiais genéticos apresentaram alguma resistência, inclusive cultivares utilizadas na olericultura fluminense, como Myr10, Myr-29 e Margareth.

Avaliações realizadas com 39 e 46 DAT, possibilitaram formação de três grupos de genótipos, quando se empregou a escala de notas, coincidindo com os resultados anteriores, nos quais as introduções BGH/UFV 5086 e 1774 foram as mais resistentes ao ácaro. No entanto, na terceira avaliação (39 DAT), a introdução BGH/UFV 1774 não diferiu da introdução BGH/UFV 1465. A intensificação dos sintomas, a partir dessa época de avaliação, deu-se aceleradamente, inclusive na introdução BGH/ UFV 1465, que não pôde mais ser considerada resistente.

Pelos dados obtidos verifica-se que da quinta avaliação em diante (53 DAT) todos genótipos avaliados, com exceção do BGH/UFV 5086, apresentaram notas elevadas, algumas já se aproximando do nível máximo de intensidade de sintomas, incluindo a até então resistente BGH/UFV 1465. Conclui-se que as condições experimentais exerceram rigorosa pressão, de tal forma que somente os materiais genéticos com alto potencial de resistência à praga conseguiram destacar-se ao final do ensaio.

Em decorrência das avaliações efetuadas foi possível constatar que a introdução BGH/UFV 2901 caracterizou-se pelo máximo grau de suscetibilidade ao ácaro branco, ao passo que BGH/UFV 5086 exibe a mais alta resistência. Já a cultivar Cascadura Ikeda apresentou o maior número de ácaros $/ \mathrm{cm}^{2}$ de área foliar, sendo, portanto, um hospedeiro que favorece a disseminação de $P$. latus e que deve ser usado para esta finalidade. A introdução BGH/UFV 5086 mostrou-se superior também nesse particular, ou seja, dificultando sua colonização pelo ácaro.

Ficou caracterizado que, dependendo do genótipo, não existe relação direta entre o número de ácaros e a intensidade dos danos. Segundo Bassett (1981) a manifestação de sintomas pode evoluir rapidamente em certos hospedeiros, onde um pequeno número de ácaros seria suficiente para provocar dano econômico.

Quando a intensidade dos sintomas aproximou-se da nota 3,0, foi observada uma diminuição de ácaros infestantes, com exceção do genótipo Myr-29 (49 DAT e 53 DAT). Essa redução já era esperada, pois folhas com sintomas severos tornam-se inadequadas ao desenvolvimento da praga, que tende a migrar para folhas mais novas ("ponteiros"). Registros semelhantes foram fei- 
tos por Chiavegato (1971) em feijoeiro. De acordo com Jeppson et al. (1975), o ácaro branco é muito exigente em termos de qualidade do substrato colonizado, mostrando acentuada preferência por tecidos novos e tenros.

A introdução BGH/UFV 5086 apresentou, em todas as épocas de avaliação, superioridade em relação aos demais materiais avaliados. A introdução BGH/ UFV 1774, apesar de mostrar um certo grau de dano, diante da extrema pressão estabelecida, não deve ser desprezada e descartada como fonte de resistência a $P$. latus, considerando-se um futuro programa de manejo integrado da praga.

A máxima intensidade de dano foi alcançada em épocas distintas comparadas da máxima infestação do ácaro ( 88 DAT e 39 DAT, respectivamente). Desse modo, ficou demonstrado que os métodos utilizados para caracterizar resistência não produzem resultados equivalentes.

Pelo que foi observado, o parâmetro mais adequado para seleção de genótipos, objetivando resistência ao ácaro branco, parece ser o da intensidade de danos causados. Segundo Gerson (1992), sintomas induzidos pelo ácaro branco podem persistir mesmo após a eliminação da praga mediante tratamentos com pesticidas.

Nas condições experimentais adotadas, apenas a introdução BGH/ UFV 5086 mostrou alto grau de resistência a $P$. latus, durante todo o período de avaliação. Torna-se necessário, contudo, avaliar esse genótipo isoladamente, situação que poderia alterar o nível de resistência, pelo fato do ácaro ficar sem opção de alimento.

Apesar da conveniência de se averiguar o desempenho de genótipos de Capsicum frente a $P$. latus em condições de campo, experimentos em casa de vegetação podem servir para predizer resultados. Vale também salientar que não se encontraram dados publicados no Brasil quanto a danos devidos a $P$. latus em lavouras de pimentão, bem como a respeito da possível resistência de qualquer das cultivares ou introduções aqui estudadas.

Os resultados presentemente relatados indicam possibilidades para o controle de $P$. latus através de programas de melhoramento que privilegiam o aumento do nível de resistência existente no germoplasma de Capsicum disponível.

\section{LITERATURA CITADA}

BASSETT, P. Observations on broad mite (Polyphagotarsonemus latus) (Acari: Tarsonemidae) attacking cucumber. Crop Protection. Conference Pest and Diseases, v. 1, p. 99-103, 1981.

VILLAS BÔAS, G.L. Métodos de controle de pragas em hortaliças. Horticultura Brasileira, Brasília, v. 7, n. 1, p. 3-6, 1989.

BROWN, R.D.; JONES, V.P. The broad mite on lemon in southern California. California Agriculture, v. 27, p. 21-22, 1983.

CHIAVEGATO, L.G. Ácaros encontrados na cultura do feijoeiro no Estado de São Paulo. In SIMPÓSIO BRASILEIRO DE FEIJÃO. Campinas: Ministério da Agricultura/Secretaria da Agricultura do Estado de São Paulo, 1971. p. 28-31.

CHIAVEGATO, L.G. Flutuação de populações de ácaros na cultura algodoeira em algumas regiões do Estado de São Paulo. Bragantia, Campinas, v. 34, n. 15, p. 241-255, 1975.

CROSS, J.V. Broad mite, Polyphagotarsonemus latus (Banks), causing damage to peppers. Plant Pathology, v. 28, p. 55, 1979.

CROSS, J.V.; BASSETT, P. Damage to tomato and aubergine by broad mite, Polyphagotarsonemus latus (Banks). Plant Pathology, v. 31, p. 391-393, 1982.

DAVID, P.M.M. Resurgence of sucking pests: In: PROCEEDINGS OF NATIONAL SYMPOSIUM. Coimbatore, India: Center for Plant Protection Studies, Tamil Nadu Agriculture University, 1986. p. 65-72.

DEPESTRE, T.; GOMEZ, O. New sweet pepper cultivars for Cuba off-season production. Capsicum and Eggplant Newsletter, v. 14, p. $47-$ 49, 1995. (Resumo)

FAN, Y., PETITT, F.L. Biological control of broad mite, Polyphagotarsonemus latus (Banks), by Neoseiulus barkeri Hughes on pepper. Biological Control, v. 4, p. 390-395, 1994.

FLECHTMANN, C.H.W. Introdução á família Tarsonemidae Kramer, 1877 (Acarina) no Estado de São Paulo. Anais da Escola Superior de Agricultura "Luiz de Queiroz", Piracicaba, v. 24, p. 265-272, 1967.

GALLO, D.; NAKANO, O.; SILVEIRA NETO, S.; CARVALHO, R.P.L.; BATISTA, G.C.;BERTI FILHO, E.; PARRA, J.R.P.; ZUCCHI, R.A.; ALVES, S.B.; VENDRAMIM, J.D. Pragas das Plantas e seu Controle. In: Manual de Entomologia Agrícola. São Paulo: Agrônomica Ceres, 1988. p. 321-613.

GERSON, U. Biology and control of the broad mite, Polyphagotarsonemus latus (Banks) (Acari: Tarsonemidae). Experimental and Applied Acarology, v. 13, p. 163-178, 1992.

GERSON, U. The broad mite, Polyphagotarsonemus latus. Phytoparasitica, v. 14, p. 157, 1986.

GIBSON, R.W.; VALENCIA, L. A survey of potato species for resistance to the mite Polyphagotarsonemus latus, with particular reference to the protection of Solanum berthaultii and S. tarijense by glandular hairs. Potato Research, v. 21, p. 217-223, 1978.

HAMBLETON, E.J. A ocorrência do ácaro tropical Tarsonemus latus Banks, (Acar. Tarsonemidae), causador da rasgadura das folhas nos algodoais de S. Paulo. Arquivos do Instituto Biológico, São Paulo, v. 9, p. 201-209, 1938.
JEPPSON, L.R.; KEIFER, H.H.;, BAKER, E.W. Mite Injures to Economic Plants. University of California Press, Berkeley, 1975. 614 p.

LARA, F.M. Princípios da Resistência de Plantas a Insetos. São Paulo: Ed. Ícone, 1991. 336 p. LI, L.S.; LI, Y.R.; BU, G.S. The effect of temperature and humidity on the growth and development of the broad mite, Polyphagotarsonemus latus. Acta Entomologica Sinica, v. 28, p. 181-187, 1985. (Resumo)

NAGAI, H.; LOURENÇÃO, A.L. Pimenta Cambuci "IAC UBATUBA". O Agronômico, Campinas, v. 39, n. 2, p. 120-121, 1987.

OLIVEIRA, C.A.L. Ação dos ácaros Polyphagotarsonemus latus (Banks, 1904) $e$ Tetranychus urticae (koch, 1936), na depreciação quantitativa e qualitativa da produção algodoeira. Piracicaba, ESALQ, 1972. 150 p. (Tese mestrado). OLIVEIRA, C.A.L.; CALCAGNOLO, G. Ação do "ácaro branco" Plolyphagotarsonemus latus (Banks, 1904) na depreciação quantitativa e qualitativa da produção algodoeira. O Biológico, São Paulo, v. 40, p. 139-149, 1974.

PARRA, J.R.P. O ácaro branco, Hemitarsonemus latus (Banks), inimigo cosmopolita de plantas cultivadas. O Agronômico, Campinas, v. 20, n. 34, p. 34-40, 1968.

PEIXOTO, J.R.; MALUF, W.R.; CAMPOS, V.P. Avaliação de genótipos de pimentão quanto à resistência a Meloydogyne incognita (raça 2) e a Meloydogyne javanica. Horticultura Brasileira, Brasília, v. 13, n. 2, p. 154-158, 1995.

PEÑA, J.E. Realationships of broad mite (Aari: Tarsonemidae) densyty to lime damage. Journal of Economic Entomology, v. 83, n. 5, p. 2008-2015, 1990.

PEÑA, J.E.; BULLOCK, R.C. Efects of feeding of broad mite (Acari:Tarsonemidae) on vegetative plant growth. Florida Entomologist, v. 77, n. 1, p. 180-184, 1994.

RAO, D.M., AHMED, K. Effect of synthetic pyrethroid and other insecticides on the resurgence of chilli yellow mite, Polyphagotarsonemus latus (Banks). In: PROCEEDINGS OF NATIONAL SYMPOSIUM. Coimbatore, India: Center for plant Protection Studies, Tamil Nadu Agricultural, University, 1986. p. 73-77.

RAO, D.M., AHMED, K. Evaluation of pesticides for the control of chilli white mite, Hemitarsonemus latus Banks (Tarsonemidae: Acarine). In: PROCEEDINGS OF NATIONAL SEMINAR ON THE PRODUCTION TECHNOLOGY OF TOMATO AND CHILLIES. Coimbatore, India: Faculty of Horticulture, Tamil Nadu Agricultural University, 1983. p. 160-162. RODITAKIS, N.E., DROSSOS, N.I. First record of Polyphagotarsonemus latus (Banks 1904) (Acari: Tarsonemidae) on greenhouse peppers in Crete. Entomologia Hellenica, v. 5, p. 35-36, 1987. SCHOONHOVEN, A.V.; PIEDRAHITA, J.; VALDERRAMA, R. et al. Biología, daño y control del ácaro tropical Polyphagotarsonemus latus (Banks) (Acarina: Tarsonemidae) en frijol. Turriabla, v. 28, n. 1, p. 77-80, 1978.

SCOTT, A.J.; KNOTT, M. A cluster analysis method for grouping means in the analysis of variance. Biometrics, v. 30, p. 507-512, 1974.

SMITH, F.F. Control of cyclamen and broad mite in gerbera, USDA, Circ. $\mathrm{n}^{\circ} .516,1939.14$ p.

VENDRAMIM, J.D. A resistência de plantas e o manejo de pragas. In: CROCOMO, W.D. ed. Manejo de Pragas: Curso de Extensão Universitária. Botucatu: FEPAF/UNESP, 1984.p. 117-146. 